\title{
Modelo DIL-D C: diagnosticando las acciones de responsabilidad social corporativa centradas en la integración laboral de personas con discapacidad
}

\author{
DIL-D () Model: a corporate social \\ responsibility diagnosis focused on the \\ employment of people with disabilities
}

\section{Resumen}

El principal objetivo de la presente investigación es analizar y

diagnosticar el grado de implantación y desarrollo de las acciones de responsabilidad social corporativa que facilitan la integración laboral de personas con discapacidad (RSC-D). Para ello hemos desarrollado un modelo clasificatorio (Modelo DIL-D (C) y un instrumento diagnóstico que dota de herramientas objetivas a los profesionales, en aras de fomentar la integración laboral de personas con discapacidad. El instrumento desarrollado se ha administrado a responsables RSC y/o de RRHH de 42 empresas. Los resultados nos muestran la confiabilidad del instrumento, así como el ajuste del Modelo. Dos tercios de las empresas obtienen niveles altos de desarrollo en todas las dimensiones, si bien un $34,5 \%$ bajos. El modelo establece las pautas de intervención que la empresa ordinaria debe seguir en aras de promover la integración laboral, facilitando las herramientas de diagnóstico que promueven la creación de entornos integradores.

\section{Palabras clave}

Responsabilidad social corporativa, políticas, sistemas de gestión, cultura, Modelo DIL-D ㄷ.

\begin{abstract}
The main objective of this research is to analyze and diagnose the degree of implementation and development of corporate social responsibility policies that facilitate the integration of people with disabilities (RSC-D). We have developed a model (DIL-D @ C Model) and a diagnostic instrument that serve as objective tools for human resources practitioners. The instrument has been administered to 42 companies, whose informants have been HR or CSR managers. The results show the internal consistency of the scales and the adjustment of the DIL-D Model $\odot$. Two-thirds of the analyzed companies obtained high levels in all dimensions, while $34.5 \%$ had low levels of development. The model establishes guidelines for interventions in the ordinary labour market in order to promote labour integration, facilitating diagnosis tools to create inclusive environments.
\end{abstract}

\section{Keywords}

Corporate social responsibility, policies, management systems, culture, DIL-D @ Model.

\author{
Marina Romeo Delgado \\ <mromeo@ub.edu> \\ Universidad de Barcelona
}

\section{Montserrat Yepes Baldó}

<myepes@ub.edu>

Universidad de Barcelona

\section{Francisco Javier Pérez \\ Conesa \\ <fcperez@ub.edu> \\ Universidad de Barcelona}

Para citar:

Romeo, M. et al. (2016): "Modelo DIL-D (): diagnosticando las acciones de responsabilidad social corporativa centradas en la integración laboral de personas con discapacidad", Revista Española de Discapacidad, 4 (I): II9-I33.

Doi: <http://dx.doi.org/IO. 5569/23405 I04.04.01.07>

Fecha de recepción: I 8-02-20I6 Fecha de aceptación: I 8-05-20I6 


\section{Introducción}

Definir conceptualmente la responsabilidad social corporativa (RSC) es una tarea compleja dada la dispersión terminológica existente en torno a este concepto: ética de los negocios (Werhane y Freeman, I999), ciudadanía empresarial (Maignan et al., I999), inversión social corporativa (Carrol, I979), gobierno corporativo (Fligstein y Feeland, I995) y sustentabilidad o sostenibilidad corporativa (Taneja et al., 20I I). No obstante, todos estos términos presentan en común el tener una marcada orientación hacia la sociedad, el medio ambiente, la ética, la gestión de stakeholders y el gobierno corporativo (Egri y Ralston, 2008; Lockett et al., 2006), además de caracterizarse por ser actuaciones no normativas sino voluntarias (Puig y Martínez, 2008).

En este sentido, el Foro de Expertos de Responsabilidad Social de las Empresas señala que:

"La Responsabilidad Social de la Empresa es, además del cumplimiento estricto de las obligaciones legales vigentes, la integración voluntaria en su gobierno y gestión, en su estrategia, políticas y procedimientos, de las preocupaciones sociales, laborales, medio ambientales y de respeto a los derechos humanos que surgen de la relación y el diálogo transparentes con sus grupos de interés, responsabilizándose así de las consecuencias y los impactos que se derivan de sus acciones (Foro de Expertos de Responsabilidad Social de las Empresas, 2007: 7).

De ahí que pueda afirmarse que la RSC consista en integrar los intereses de todos los individuos o stakeholders en las políticas y acciones de la organización. En concreto, la Comisión Europea (20II) establece como expresiones de RSC el conjunto de políticas relacionadas con los derechos humanos, las prácticas de trabajo y de empleo, las cuestiones medioambientales y la lucha contra el fraude y la corrupción, la participación de las comunidades locales y su desarrollo, la integración de las personas con discapacidad y los intereses de los consumidores y la divulgación de información no financiera y el voluntariado entre los trabajadores.

A todas estas acciones de carácter voluntario cabe añadir las desarrolladas desde el ordenamiento jurídico español. En concreto, en el marco de la integración de las personas con discapacidad se ha venido realizando una clara apuesta respecto a la reserva de puestos de trabajo para personas con discapacidad, especialmente desde la aprobación de la Ley I3/I982, de 7 de abril, de integración social de los minusválidos (LISMI), y recientemente con la aprobación del Texto Refundido de la Ley General de derechos de las personas con discapacidad y de su inclusión social (Real Decreto Legislativo I/2013, de 29 de noviembre).

Si bien el desarrollo del marco legal y el despliegue de las políticas de RSC han supuesto un cambio positivo en la visión de las empresas respecto de las personas con discapacidad (Alfaro Faus y Vallés López, 20I 5), la integración laboral de este colectivo y su participación efectiva en el mercado de trabajo continúa mostrando problemáticas específicas y disfuncionalidades (Romeo et al., 20I6). Muestra de ello es cómo en España, y según datos del Servicio Estatal de Empleo (SEPE, 2OI 5), el 37,4\% de las personas con discapacidad legalmente reconocida eran activos en 2013 , siendo esta tasa de actividad casi 40 puntos inferior a la de la población sin discapacidad $(77,2 \%)$.

A todo ello cabe añadir que existen pocos estudios que se preocupen por el análisis de dichas políticas, siendo la mayoría de ellos informes técnicos desarrollados por las propias organizaciones. Algunos ejemplos en este sentido es el informe técnico de Pizza Hut, en el que se señala que la tasa de retención de empleados con discapacidad es un $72 \%$ más alto que la de los demás empleados, haciendo referencia también al ahorro para la organización de millones de dólares, cada año, en costes de reclutamiento y capacitación (National Disability Coordination 
Officer Program, s.f.). Asimismo, un informe de Carolina Fine Snacks constata que la rotación se redujo del $80 \%$ a menos del $5 \%$, la productividad aumentó del $70 \%$ al $95 \%$, y el absentismo disminuyó del $20 \%$ a menos del $5 \%$ tras la incorporación de diez personas con discapacidad, lo que suponía la mitad de su plantilla (Hogan, 2003).

En España, existen también algunos ejemplos de buenas prácticas en relación a la inclusión de personas con discapacidad, tal y como podemos constatar en Cortés Ortiz (20I I). El estudio incluye un análisis de las memorias de RSC de las empresas participantes en el Ibex 35, de las cinco mejores empresas del Great Place to Work Institute en 2009 y de tres grandes corporaciones españolas presentes en el ranking de la revista Fortune, y concluye que, en general, los proyectos que se desarrollan en materia de integración tienen un impacto positivo en el desempeño laboral y en el compromiso. No obstante, se plantean importantes limitaciones dado que:

\footnotetext{
"no hay planes globales de acción en discapacidad dentro de las políticas de RSE de las empresas, ni a medio ni a largo plazo, por lo que los proyectos que se desarrollan son esporádicos, es decir, no presentan una continuidad en el tiempo" (Cortés Ortiz, 2OI I: IO2).
}

A ello cabe añadir que el conjunto de estudios desarrollados indican que las acciones implementadas no se hallan alineadas con la estrategia de la organización. Por ello, se convierten en acciones técnico-operativas que:

a. no permiten minimizar o corregir el impacto negativo de determinadas intervenciones.

b. no dotan a los responsables de recursos humanos y de RSC de herramientas de diagnóstico de las políticas de integración ni de pautas para la intervención.

Tal y como señala el Libro Verde de la Comisión Europea (200I), en aquellas empresas con mayor grado de implantación y desarrollo de dichas políticas:

\begin{abstract}
"se pueden derivar resultados positivos directos de, por ejemplo, un mejor entorno de trabajo - que genere un mayor compromiso de los trabajadores e incremente su productividad - o de una utilización eficaz de los recursos naturales. Además, se logran efectos indirectos a través del aumento de la atención que prestan a la empresa consumidores e inversores, que ampliará sus posibilidades en el mercado" (Comisión Europea, 200I: 8).
\end{abstract}

En este contexto se nos hace del todo necesario el desarrollo de la presente investigación, cuyo objetivo es analizar y diagnosticar el grado de implantación y desarrollo de las políticas y estrategias de responsabilidad social corporativas que facilitan la integración laboral de personas con discapacidad.

Para ello hemos considerado oportuno, en primer lugar, desarrollar un modelo clasificatorio (Modelo DIL-D (C) y un instrumento diagnóstico (RSC-D), que permita evaluar y diagnosticar el grado de despliegue de las políticas y estrategias de integración laboral de personas con discapacidad en la empresa ordinaria.

\section{EI Modelo DIL-D @}

Para el diseño del Modelo, siguiendo a la Fundación ONCE (2009) y a la Fundación Bequal (20II) - integrada por el Comité Español de Representantes de Personas con Discapacidad (CERMI), Fundación ONCE, FEACEM, la Fundación Seeliger y Conde Diversity - , hemos estructurado el conjunto de acciones orientadas a la RSC-D en cuatro grandes dimensiones:

- Políticas: Referidas a todas aquellas directrices, reglas y procedimientos establecidos por la organización con el propósito de apoyar los esfuerzos para lograr los objetivos previamente planificados 
(Fred, 2003). Incluye diversos aspectos relacionados con los niveles de análisis interno de la discapacidad e integración a partir de las encuestas de clima, los planes de normalización y de acción social desarrollados, la adaptación de los planes de comunicación, las alianzas estratégicas con entidades especializadas en integración de personas con discapacidad y el compromiso corporativo con la integración.

- Sistemas de gestión: Acciones desarrolladas relativas a la disponibilidad y utilización efectiva de las políticas impulsadas por la organización a través de los procesos de selección e incorporación, formación, desarrollo profesional, prevención de riesgos, adaptación del entorno de trabajo y comunicación (Quijano et al., 2006).

- Facilitadores: Todos aquellos elementos de la organización que permiten y fortalecen el desarrollo de las políticas de RSC-D (Carlier et al., 2012). Dichos elementos se relacionan principalmente con el grado de implicación de los stakeholders (empleados, equipo directivo, personas con discapacidad, sus familiares, administración pública, sindicatos, tejido asociativo, $\mathrm{y}$ comunidad local). Entre estos tienen un papel especial los directivos y gerentes organizacionales, tanto a la hora de desarrollar políticas integradoras como facilitando la identificación, el compromiso, la satisfacción laboral y la efectividad de los empleados (Quijano et al., 2008; Veríssimo y Lacerda, 20I 5 ).

- Cultura: Definida como el conjunto de valores, creencias, estilos de trabajo y relaciones que distinguen a una organización de otra (Harrison, I972), lo que incluye el compromiso de la dirección con la RSC-D, beneficios y objetivos corporativos e indicadores clave de desempeño (KPI's) en materia de discapacidad.

De estas cuatro, las políticas y la cultura representan una dimensión estratégica de la intervención, es decir, aquellos aspectos vinculados al "hacia dónde va la organización". En cambio, los sistemas de gestión y los facilitadores atienden a la vertiente más operativa, esto es, a los elementos que la empresa "debe poner en marcha" para alcanzar los objetivos establecidos. Ambos aspectos, estratégicos y operativos deben ser tenidos en cuenta a la hora de analizar el grado de despliegue de las políticas de integración laboral de personas con discapacidad, de ahí que sean considerados en el instrumento diagnóstico (RSC-D).

En la Tabla I presentamos una comparativa de las dimensiones planteadas por ambos modelos en relación a nuestra propuesta.

En resumen, nuestro modelo resulta un avance teórico y metodológico, tal y como ratificarán los resultados que a continuación presentamos, en relación a sus predecesores, ya que además de integrar las propuestas conceptuales de ONCE y Bequal, incluye por un lado, el papel clave de los stakeholders, definidos en términos de la International Organization for Standardization (ISO, 20IO) y las normas AAIooo y SA 8000 (AccountAbility, 20I I ISO, 20IO), y por otro el de la cultura organizacional entendida como el modo de funcionamiento organizativo sobre el que se estructura la estrategia organizativa y los sistemas de gestión de la misma. Además, al tener en cuenta la perspectiva estratégica y operativa, genera una visión más sistémica de la realidad organizacional.

\section{Método}

\section{Procedimiento}

Partiendo de la información disponible en la base de datos SABI (Sistema de Análisis de Balances Ibéricos), se realizó una selección de empresas, teniendo en cuenta los siguientes criterios e indicadores de inclusión: 
Tabla 1. Comparativa de los modelos de evaluación de la RSC-D en ONCE, Fundación Bequal, y Modelo DIL-D $\odot$

\begin{tabular}{|c|c|c|}
\hline ONCE & Fundación Bequal & Modelo DIL-D @ \\
\hline Código ético & Estrategia y liderazgo & Cultura \\
\hline $\begin{array}{l}\text { Sensibilización y formación } \\
\text { Contratación de personas con discapacidad } \\
\text { Gestión de RRHH (reclutamiento, selección, contratación, } \\
\text { acogida, promoción, formación, prevención de riesgos } \\
\text { laborales) } \\
\text { Formación del personal de atención al cliente para la } \\
\text { atención de personas con discapacidad }\end{array}$ & $\mathrm{RRHH}$ & \multirow[t]{2}{*}{ Sistemas de gestión } \\
\hline Actuaciones de mejora de la accesibilidad & Accesibilidad & \\
\hline $\begin{array}{l}\text { Política o directriz orientada a considerar o favorecer a los } \\
\text { proveedores y/o contratistas que empleen a personas con } \\
\text { discapacidad, incluidos los Centros Especiales de Empleo }\end{array}$ & Compra responsable & $\begin{array}{l}\text { Cumplimiento de } \\
\text { medidas alternativas a } \\
\text { la LISMI }\end{array}$ \\
\hline $\begin{array}{l}\text { Productos y/o servicios que incorporan los principios de } \\
\text { diseño para todos y accesibilidad universal }\end{array}$ & Clientes & - \\
\hline $\begin{array}{l}\text { Actuaciones consolidadas en el ámbito de la acción } \\
\text { social, orientadas parcial o totalmente a las personas con } \\
\text { discapacidad (patrocinios, donaciones, financiación de } \\
\text { proyectos, programas de voluntariado...) } \\
\text { Contacto establecido con organizaciones representativas } \\
\text { de las personas con discapacidad en el proceso de } \\
\text { consulta con los grupos de interés }\end{array}$ & $\begin{array}{l}\text { Acción social } \\
\text { Estrategia y liderazgo }\end{array}$ & Políticas \\
\hline- & Comunicación & $\begin{array}{l}\text { Políticas } \\
\text { Sistemas de gestión }\end{array}$ \\
\hline- & Estrategia y liderazgo & Facilitadores \\
\hline
\end{tabular}

Fuente: elaboración propia.

- $\quad$ Empresas de capital privado.

- $\quad$ Susceptibles de aplicación de la LISMI (con un número de empleados superior a 50 ).

- Estabilidad en el mercado: empresas activas y antigüedad mínima de 7 años bajo el mismo NIF.

- Exclusión de los grupos empresariales: disponibilidad de cuentas anuales no consolidadas.
La selección de las empresas que conforman la muestra se ha realizado a partir de un muestreo accidental.

\section{Participantes}

Los participantes son responsables de recursos humanos y/o de RSC de las empresas 
seleccionadas. En total se han realizado 42 entrevistas, si bien el total de cuestionarios completos es de 39. En la Tabla 2 se presentan las características de las empresas participantes.

\begin{tabular}{|c|c|c|}
\hline \multicolumn{3}{|c|}{$\begin{array}{l}\text { Tabla 2. Características de las empresas } \\
\text { participantes }\end{array}$} \\
\hline \multicolumn{2}{|c|}{$\begin{array}{l}\text { Características de las empresas } \\
\text { participantes }\end{array}$} & \multirow{2}{*}{$\begin{array}{r}\% \\
62,3 \\
\end{array}$} \\
\hline Distribución por género & Hombres & \\
\hline de los empleados & Mujeres & 37,9 \\
\hline \multirow{4}{*}{ Ámbito de actuación } & Internacional & 50 \\
\hline & Nacional & 32,5 \\
\hline & Regional & 5 \\
\hline & Local & 12,5 \\
\hline \multirow{8}{*}{ Sector empresarial } & $\begin{array}{l}\text { Industria } \\
\text { manufacturera }\end{array}$ & 56,4 \\
\hline & Construcción & 2,6 \\
\hline & $\begin{array}{l}\text { Comercio al } \\
\text { por mayor } \\
\text { y al menor, } \\
\text { reparación de } \\
\text { vehículos } \\
\end{array}$ & 5,1 \\
\hline & $\begin{array}{l}\text { Transporte y } \\
\text { almacenamiento }\end{array}$ & 5,1 \\
\hline & $\begin{array}{l}\text { Actividades } \\
\text { profesionales, } \\
\text { científicas y } \\
\text { técnicas } \\
\end{array}$ & 10,3 \\
\hline & \begin{tabular}{|l} 
Actividades \\
administrativas \\
y servicios \\
auxiliares \\
\end{tabular} & 5,1 \\
\hline & Hostelería & 10,3 \\
\hline & Otros servicios & 5,1 \\
\hline \multicolumn{2}{|c|}{$\begin{array}{l}\text { Empresas que cumplen con el } 2 \% \text { de } \\
\text { trabajadores con discapacidad }\end{array}$} & 79,8 \\
\hline \multirow{4}{*}{$\begin{array}{l}\text { Empresas que cumplen } \\
\text { con medidas alternativas }\end{array}$} & \begin{tabular}{|l} 
Donación o \\
patrocinio
\end{tabular} & 11,9 \\
\hline & $\begin{array}{l}\text { Contratación } \\
\text { con un CEE }\end{array}$ & 21,4 \\
\hline & No se aplican & 50 \\
\hline & NS/NC & 7,1 \\
\hline
\end{tabular}

Fuente: elaboración propia.

\section{Instrumento}

Para el desarrollo del instrumento RSC-D se reunió un grupo de expertos del ámbito de la integración laboral para que analizaran los aspectos legales, psicosociales y económicos que deberían tenerse en cuenta a la hora de evaluar la responsabilidad social en materia de integración laboral de personas con discapacidad.

Desarrollada una primera propuesta, se procedió al redactado de los ítems que deberían configurar cada una de las dimensiones anteriormente descritas: políticas, sistemas de gestión, facilitadores y cultura. El instrumento así creado fue sometido a una prueba piloto con otros siete expertos de la temática para garantizar la correcta comprensión de sus ítems, independientemente del sector y del tipo de empresa. Se procedió a reajustar el redactado de los ítems siguiendo las indicaciones del grupo de expertos.

El instrumento final se compone de 32 ítems donde se combinan preguntas dicotómicas (I I ítems) y tipo Likert (2 I ítems) organizadas en las cuatro dimensiones:

- Políticas (6 ítems): Incluye diversos aspectos relacionados con la evaluación de las percepciones de los empleados sobre las políticas de discapacidad, la existencia de planes de normalización, acción social, planes de comunicación, alianzas y compromiso corporativo. Un ejemplo de ítem es "Se colabora con la Comunidad Local y el tejido asociativo de la discapacidad para sensibilizar a la sociedad e impulsar la incorporación e integración de personas con discapacidad en el mercado laboral". La escala de medida es una escala Likert de 4 puntos $(\mathrm{I}=\mathrm{No}, 2=\mathrm{En}$ proyecto, $3=$ En Desarrollo, 4=Implementado).

- Sistemas de gestión ( I I ítems): Evalúa, en una escala dicotómica ( $\mathrm{I}=\mathrm{Si}, 2=\mathrm{No}$ ), si la organización ha adaptado los sistemas de selección e incorporación, formación, 
desarrollo profesional, prevención de riesgos, adaptación del entorno de trabajo y comunicación a las necesidades específicas de las personas con discapacidad.

- Facilitadores (I I ítems): Evalúa el grado de implicación de los stakeholders (empleados, equipo directivo, personas con discapacidad y sus familias, administración pública, sindicatos, tejido asociativo, y comunidad local) en las diferentes iniciativas de integración laboral de personas con discapacidad puestas en marcha por la organización ( 8 ítems). Por otro lado, se analiza el grado en que los directivos están sensibilizados y son ejemplo de conducta integradora ( 3 ítems). Ambas dimensiones se miden en una escala Likert de 4 puntos ( $\mathrm{I}=$ Nada, 2=Poco, 3 =Bastante, 4=Mucho).

- Cultura (4 ítems): Analiza el grado en que la organización tiene descritos objetivos corporativos e indicadores clave de desempeño (KPI's) en materia de discapacidad. Se mide en una escala Likert de 4 puntos ( $\mathrm{I}=\mathrm{Nada}, 2=\mathrm{Poco}, 3=$ Bastante, 4=Mucho).

\section{Análisis de datos}

El análisis de datos se realizó utilizando el software PASW Statistics 2I. En primer lugar se analizó la confiabilidad de las escalas a partir del a de Cronbach y Kuder-Richardson 20 (KR2O) (para las escalas dicotómicas). Posteriormente se realizaron correlaciones entre las escalas. Finalmente se calculó la puntuación por dimensiones y se ajustó el Modelo Clasificatorio del Grado de Desarrollo de las Políticas y Estrategias de Integración Laboral de Personas con Discapacidad (DIL-D (C) a partir de la combinación de las 4 dimensiones.

\section{Resultados}

Para dotar de mayor claridad expositiva a los resultados, en primer lugar, presentamos el grado de confiabilidad de las escalas del instrumento RSC-D. Posteriormente mostramos los estadísticos descriptivos de cada una de las dimensiones que configuran el instrumento y finalizamos con el ajuste del Modelo Clasificatorio DIL-D @ .

\section{Confiabilidad: a de Cronbach y Kuder- Richardson 20 (KR20)}

La Tabla 3 muestra el grado de confiabilidad de cada una de las escalas que configuran el cuestionario. Como se observa, el nivel de confiabilidad de acuerdo al coeficiente alfa de Cronbach y Kuder-Richardson 20 varía en las distintas dimensiones entre .8 I I y .903, lo que indica el alto grado de confiabilidad de las mismas.

\begin{tabular}{l}
\hline Tabla 3. Confiabilidad de las escalas \\
\begin{tabular}{|l|c|}
\hline Dimensión & $\begin{array}{c}\alpha \text { de Cronbach / } \\
\text { Kuder-Richardson 20 }\end{array}$ \\
\hline Políticas & 0,833 \\
\hline Sistemas de gestión & 0,811 \\
\hline Facilitadores & 0,903 \\
\hline Cultura & 0,854 \\
\hline
\end{tabular}
\end{tabular}

Fuente: elaboración propia.

\section{Análisis descriptivo por dimensión}

A continuación, presentamos los análisis descriptivos atendiendo a cada una de las cuatro dimensiones del modelo. En primer lugar, mostramos los resultados descriptivos de la dimensión sobre políticas (Tabla 4). Como se observa, las políticas que poseen el mayor nivel de desarrollo en las empresas son las que están relacionadas con la colaboración con la comunidad local y el tejido asociativo, así como 


Tabla 4. Estadísticos descriptivos de la dimensión sobre políticas
\begin{tabular}{|l|l|l|l|}
\hline Política & Media & Desv. típ. & $\begin{array}{c}\% \text { de empresas que tienen } \\
\text { la } \\
\text { implica en desarrollo o } \\
\text { imtada }\end{array}$ \\
\hline $\begin{array}{l}\text { Evaluación de las percepciones de los empleados sobre } \\
\text { las políticas de discapacidad }\end{array}$ & 1,58 & 1,083 & $19 \%$ \\
\hline $\begin{array}{l}\text { Colaboración con la Comunidad Local y el tejido } \\
\text { asociativo }\end{array}$ & 2,74 & 1,380 & \\
\hline $\begin{array}{l}\text { Adaptación del plan de comunicación a las necesidades } \\
\text { específicas de los empleados }\end{array}$ & 1,86 & 1,181 & $61,9 \%$ \\
\hline $\begin{array}{l}\text { Desarrollo de alianzas con entidades especialistas en } \\
\text { integración de personas con discapacidad }\end{array}$ & 2,59 & 1,396 & $33,3 \%$ \\
\hline $\begin{array}{l}\text { Existencia de políticas corporativas que definen el } \\
\text { compromiso y los objetivos de la compañía en materia de } \\
\text { integración laboral de personas con discapacidad }\end{array}$ & 2,29 & 1,293 & $52,4 \%$ \\
\hline $\begin{array}{l}\text { Existencia de un plan de normalización de la discapacidad } \\
\text { en el entorno empresarial y laboral }\end{array}$ & 1,76 & 1,206 & \\
\hline
\end{tabular}

el desarrollo de alianzas estratégicas. En cambio, las acciones vinculadas a la evaluación de las percepciones de los empleados sobre las políticas de discapacidad es la que obtiene niveles más bajos de desarrollo (tan solo un I9\% de las empresas tienen planificadas o realizan este tipo de acciones).

En cuanto a los sistemas de gestión, en la Tabla 5 se presentan las frecuencias de respuesta para los ítems. Como se puede observar, los sistemas de gestión interna que poseen un mayor grado de despliegue en las empresas participantes son el de prevención de riesgos y adaptación del entorno laboral. Por el contrario, los que muestran un menor nivel de despliegue son los que se refieren al desarrollo profesional o plan de carrera y al desarrollo de planes de formación que respondan a las necesidades de las personas con discapacidad.

En cuanto a la dimensión que analiza los elementos que facilitan la integración laboral de personas con discapacidad vemos que el stakeholder al que se percibe con un mayor grado de implicación en las diferentes iniciativas puestas en marcha en las empresas son las propias personas con discapacidad $(68,4 \%)$, seguidos del equipo directivo $(60 \%)$ y el conjunto de empleados $(54,8 \%)$. Destaca el bajo nivel de implicación percibido de los sindicatos $(69,7 \%$, de los que un $42,4 \%$ consideran que no están nada implicados y otro $27,3 \%$ en poca medida), seguidos por las familias de los empleados $(68,6 \%)$ y la comunidad local $(64,5 \%)$ (Tabla 6).

Tabla 5. Frecuencias relativas para los ítems de la dimensión sobre sistemas de gestión

\begin{tabular}{|l|c|c|}
\hline Sistema de gestión & Adaptado & $\begin{array}{c}\text { No } \\
\text { adaptado }\end{array}$ \\
\hline Selección e incorporación & $43,2 \%$ & $56,8 \%$ \\
\hline Formación & $41,7 \%$ & $58,3 \%$ \\
\hline Desarrollo profesional & $19,5 \%$ & $80,5 \%$ \\
\hline Prevención de riesgos & $72,5 \%$ & $27,5 \%$ \\
\hline Adaptación del entorno & $72,1 \%$ & $27,9 \%$ \\
\hline Comunicación interna & $44 \%$ & $56 \%$ \\
\hline
\end{tabular}

Fuente: elaboración propia. 


\begin{tabular}{l}
\hline Tabla 6. Grado de implicación de los stakeholders \\
\begin{tabular}{|l|c|c|}
\hline Stakeholders & Media & Desv. típ. \\
\hline Empleados & 2,52 & 1,131 \\
\hline Equipo directivo & 2,78 & 1,121 \\
\hline Personas con discapacidad & 3,03 & 1,197 \\
\hline Familia & 2,09 & 1,121 \\
\hline Administración & 2,08 & 0,954 \\
\hline Sindicatos & 2,06 & 1,144 \\
\hline Tejido asociativo & 2,13 & 1,056 \\
\hline Comunidad local & 2,10 & 1,076 \\
\hline
\end{tabular}
\end{tabular}

Fuente: elaboración propia.

\begin{tabular}{l}
\hline Tabla 7. Rol de los directivos \\
\begin{tabular}{|l|c|c|c|c|c|c|}
\hline Ítem & Nada & Poco & Bastante & Mucho & Media & Desv. típ. \\
\hline $\begin{array}{l}\text { Grado de conocimiento de las políticas } \\
\text { de integración laboral de personas con } \\
\text { discapacidad de los directivos }\end{array}$ & $15,4 \%$ & $28,2 \%$ & $17,9 \%$ & $38,5 \%$ & 2,79 & 1,128 \\
\hline $\begin{array}{l}\text { Grado de sensibilización de los } \\
\text { directivos respecto a la integración } \\
\text { laboral de personas con discapacidad }\end{array}$ & $20 \%$ & $27,5 \%$ & $12,5 \%$ & $40 \%$ & 2,73 & 1,198 \\
\hline $\begin{array}{l}\text { Grado de ejemplificación de conducta } \\
\text { integradora de los directivos }\end{array}$ & $15 \%$ & $35 \%$ & $22,5 \%$ & $27,5 \%$ & 2,63 & 1,055 \\
\hline
\end{tabular}
\end{tabular}

Fuente: elaboración propia.

Tabla 8. Frecuencias y puntuaciones medias de la dimensión sobre cultura
\begin{tabular}{|l|c|c|c|c|c|c|}
\hline Ítem & Nada & Poco & Bastante & Mucho & Media & Desv. típ. \\
\hline Liderazgo y compromiso & $23,8 \%$ & $9,5 \%$ & $31 \%$ & $37,7 \%$ & 2,79 & 1,180 \\
\hline Beneficios corporativos & $51,2 \%$ & $14,6 \%$ & $19,5 \%$ & $14,6 \%$ & 1,98 & 1,151 \\
\hline Objetivos corporativos & $57,5 \%$ & $17,5 \%$ & $7,5 \%$ & $17,5 \%$ & 1,85 & 1,167 \\
\hline Key Performance Indicators (KPI's) & $65 \%$ & $5 \%$ & $7,5 \%$ & $22,5 \%$ & 1,88 & 1,285 \\
\hline
\end{tabular}

Fuente: elaboración propia.

En cuanto al rol de los directivos se constata que al menos en un $50 \%$ de las empresas participantes estos conocen las políticas, están sensibilizados y son ejemplo de conducta integradora (Tabla 7).

Por último, en relación a la cultura, alrededor del $60 \%$ de las empresas indican que la dirección conoce y apoya de forma activa las políticas en materia de discapacidad e integración. Este resultado no se corresponde con las acciones relacionadas con beneficios, objetivos corporativos e indicadores clave de desempeño (KPI's) específicos para el colectivo de empleados con discapacidad (un $69 \%$, un $65 \%$ y un $70 \%$ de las empresas respectivamente afirman que estos aspectos están poco o nada desarrollados) (Tabla 8). 


\section{Ajuste del Modelo DIL-D @}

Para desarrollar el ajuste del modelo clasificatorio se han seguido los siguientes pasos:

I. Obtención de la puntuación global bruta para cada una de las cuatro dimensiones (políticas, sistemas de gestión, facilitadores y cultura) y recategorización de las mismas en tres niveles de desarrollo (nivel bajo, medio y alto). Con objeto de ajustar el modelo clasificatorio a partir de escalas distintas de medida, se ha seguido una estrategia de estandarización de las mismas. Para ello se ha obtenido el sumatorio de las puntuaciones de las componentes y posteriormente se ha convertido a escala I-3 ( I-Nivel bajo, 2-Nivel medio, 3-Nivel alto). Ello nos permite hacer comparables diferentes escalas y organizaciones.

2. Análisis de la relación existente entre los niveles de desarrollo de las dimensiones estratégicas (políticas-cultura) y operativas (sistemas de gestión-facilitadores).

En la Tabla 9 se evidencia cómo los componentes que configuran la escala estratégica (cultura y políticas) se hallan íntimamente relacionados. Aquellas organizaciones con una cultura que no se preocupa especialmente por la promoción de la integración laboral muestran a su vez un bajo nivel de despliegue en políticas de integración laboral de personas con discapacidad. En el otro extremo, las empresas con destacados niveles de preocupación por la integración tienen un alto grado de despliegue de sus políticas de integración.

Resulta interesante reseñar que aquellas empresas con un nivel intermedio en cultura organizacional, esto es, no tan claramente orientada a la integración laboral de personas con discapacidad, presentan un nivel medio/alto de despliegue de políticas de integración.

Con respecto a la dimensión operativa, en primer lugar obtuvimos la puntación media en cuanto a la sensibilidad del equipo directivo en materia de discapacidad y su actuación como modelo de conducta integradora. Esta puntuación fue usada como coeficiente modulador de corrección de la escala de implicación del equipo directivo, minimizándose así los posibles efectos de deseabilidad social (Lavrakas, 2008).

Una vez obtenida la puntuación global vinculada al equipo directivo, hemos analizado su relación con los sistemas de gestión. Los resultados obtenidos nos muestran que altos niveles en la dimensión de facilitadores vinculados al equipo directivo coinciden con niveles altos de desarrollo en los sistemas de gestión. Por el contrario, niveles medio/bajos de desarrollo de estos facilitadores se corresponde con niveles medio/bajos en el desarrollo de los sistemas de gestión (Tabla ro).

Una vez confirmadas las relaciones entre las escalas que conforman las dimensiones operativa y estratégica se procedió al ajuste del modelo clasificatorio DIL-D @ C. Para ello se recategorizaron las diferentes combinaciones posibles entre escalas como se muestra en la Tabla I I.

Tabla 9. Relación entre las dimensiones estratégicas organizacionales (los valores corresponden a los porcentajes de columna)

\begin{tabular}{|c|c|c|c|c|}
\hline & & \multicolumn{3}{|c|}{ Cultura } \\
\hline & & Bajo & Medio & Alto \\
\hline \multirow{3}{*}{ Políticas } & Bajo & $80 \%$ & $15,4 \%$ & $7,7 \%$ \\
\hline & Medio & $10 \%$ & $38,5 \%$ & $38,5 \%$ \\
\hline & Alto & $10 \%$ & $46,1 \%$ & $53,8 \%$ \\
\hline
\end{tabular}

Fuente: elaboración propia. 
Tabla 10. Relación entre las dimensiones operativas organizacionales (los valores corresponden a los porcentajes de columna)

\begin{tabular}{|c|c|c|c|c|}
\hline & & \multicolumn{3}{|c|}{ Facilitadores vinculados al equipo directivo } \\
\hline & & Bajo & Medio & Alto \\
\hline \multirow{3}{*}{ Sistemas de gestión } & Bajo & $41,7 \%$ & $44,4 \%$ & $25,0 \%$ \\
\hline & Medio & $41,7 \%$ & $33,3 \%$ & $33,3 \%$ \\
\hline & Alto & $16,6 \%$ & $22,2 \%$ & $41,7 \%$ \\
\hline
\end{tabular}

Fuente: elaboración propia.

Tabla 11. Recategorización de las combinaciones entre las diferentes escalas

\begin{tabular}{|l|l|l|}
\cline { 2 - 3 } \multicolumn{1}{c|}{} & Nivel alto $(\mathbf{A})$ & Nivel bajo $(\mathbf{B})$ \\
\hline Estratégico & $\begin{array}{l}\text { Política y Cultura } \geq 2 \\
\text { Política }=1 \text { y Cultura }=3\end{array}$ & $\begin{array}{l}\text { Política y Cultura }=1 \\
\text { Política }=2 \text { y Cultura }=1 \\
\text { Política }=1 \text { y Cultura }=2 \\
\text { Política }=3 \text { y Cultura }=1\end{array}$ \\
\hline Operativo & $\begin{array}{l}\text { Sistemas de gestión y Facilitadores } \geq 2 \\
\text { Sistemas de gestión }=1 \text { y Facilitadores }=3\end{array}$ & $\begin{array}{l}\text { Sistemas de gestión y Facilitadores }=1 \\
\text { Sistemas de gestión }=2 \text { y Facilitadores }=1 \\
\text { Sistemas de gestión = 1 y Facilitadores }=2 \\
\text { Sistemas de gestión = 3 y Facilitadores }=1\end{array}$ \\
\hline
\end{tabular}

Fuente: elaboración propia.

En términos generales, las puntuaciones con valor igual a tres han sido etiquetadas como puntuaciones altas y las puntuaciones con valora igual a uno como bajas. No obstante, cuando en una empresa aparecen combinadas dos dimensiones con puntuaciones extremas (I-3 ó 3-I) se han categorizado como altas o bajas en función del peso otorgado a las dimensiones. De este modo, en el caso de la combinación política/cultura (dónde se da un 5,6\% de casos con puntuaciones extremas contrarias), se ha considerado dar mayor peso a la dimensión cultura en base a los planteamientos teóricos de Schein (2010), al vincularse con los aspectos más profundos de la organización, sus valores.

En el caso de la combinación sistemas de gestión/facilitadores, dónde existen un I4,2 \% de casos con puntuaciones extremas contrarias, se ha dado mayor peso a la función del equipo directivo como facilitador dado que su rol de liderazgo formal resulta clave a la hora de desarrollar e implementar políticas de integración (Waldman y Siegel, 2008) y sistemas de gestión adaptados a las mismas.

Por último, para ajustar el modelo clasificatorio se combinaron los dos niveles de estrategia con los dos operativos para obtener cuatro categorías clasificatorias de las empresas participantes según su grado de desarrollo de las políticas y estrategias de integración laboral de personas con discapacidad (Tabla I2).

Tabla 12. Clasificación de las empresas en función de su grado de desarrollo de políticas y estrategias de integración laboral de las personas con discapacidad

\begin{tabular}{|l|c|c|c|}
\hline \multicolumn{2}{|c|}{} & \multicolumn{2}{c|}{ Dimensión estratégica } \\
\cline { 3 - 4 } \multicolumn{2}{|c|}{} & Alta & Baja \\
\hline \multirow{3}{*}{$\begin{array}{l}\text { Dimensión } \\
\text { operativa }\end{array}$} & Alta & AA & BA \\
& Baja & $51,7 \%$ & $0 \%$ \\
\cline { 2 - 4 } & AB & BB \\
& $13,8 \%$ & $34,5 \%$ \\
\hline
\end{tabular}


El modelo tiene cuatro niveles de desarrollo de las políticas y estrategias de integración laboral de personas con discapacidad, denominados AA, $\mathrm{AB}, \mathrm{BA}, \mathrm{y} \mathrm{BB}$. Entre las empresas participantes encontramos un $5 \mathrm{I}, 7 \%$ con altos niveles de desarrollo estratégico y operativo (AA), un I $3,8 \%$ con alto nivel de desarrollo estratégico pero bajo nivel de desarrollo operativo $(\mathrm{AB})$ y un $34,5 \%$ con bajos niveles de desarrollo estratégico y operativo. Ninguna de las empresas participantes se sitúa en bajos niveles de desarrollo estratégico combinado con altos niveles operativos (BA).

\section{Discusión}

El objetivo de la presente investigación ha sido analizar y diagnosticar la responsabilidad social corporativa centrada en la integración laboral de personas con discapacidad (RSC-D), a partir del desarrollo de un modelo clasificatorio (Modelo DIL-D (C) y un instrumento diagnóstico (RSC-D).

El Modelo DIL-D ( $)$ permite clasificar a las organizaciones en función de cuatro niveles de desarrollo de su RSC-D (AA, AB, BA, y BB) a partir de combinar el grado de despliegue del nivel estratégico y operativo para la integración laboral de personas con discapacidad. Los análisis realizados constatan la fiabilidad y validez del instrumento RSC-D así como el ajuste del Modelo clasificatorio DIL-D @ .

El modelo DIL-D (C) se asienta en cuatro dimensiones: políticas, sistemas de gestión, facilitadores y cultura. En cuanto a las políticas, los resultados descriptivos indican que las más desarrolladas son aquellas relacionadas con la colaboración con la comunidad local y el tejido asociativo y la creación de alianzas estratégicas, mientras que las acciones vinculadas al desarrollo de políticas internas de la organización son las que están menos implementadas.
Los resultados vinculados a los sistemas de gestión muestran cómo aquellos que tienen un mayor nivel de despliegue se corresponden con los que tienen un fuerte vínculo de obligación legal de cumplimiento. Por el contrario, aquellos sistemas de gestión que no poseen dicha relación con el aspecto legal y son, más bien, de carácter voluntario, tienen un menor despliegue. En este sentido, los sistemas de selección, prevención de riesgos y adaptación del entorno son aquellos cuyo desarrollo está en mayor medida relacionado con los requerimientos legales, de ahí que su adaptación esté más desarrollada. En cambio, los sistemas de formación, desarrollo profesional y comunicación no están explicitados en la ley, de ahí que su adaptación, al ser de carácter voluntario, dote de una mayor calidad a las organizaciones que los implementan.

Si bien los resultados indican que los stakeholders más implicados en la integración laboral de personas con discapacidad son las propias personas con discapacidad, siguiendo a Waldman y Siegel (2008) consideramos que la implicación del equipo directivo resulta clave a la hora de desarrollar e implementar políticas de integración. Por ese motivo, el Modelo DIL-D (C) pondera las puntuaciones obtenidas en cuanto al liderazgo en esta dimensión, minimizándose además la posible deseabilidad social en las respuestas emitidas (Lavrakas, 2008), esto es, la tendencia a responder en base a lo que es valorable socialmente, dando una imagen sesgada de la propia organización.

Por último, en cuanto a la cultura se constata cierta incongruencia entre el nivel de conocimiento y apoyo activo por parte de la dirección de las políticas en materia de discapacidad e integración, y la concreción en objetivos corporativos (KPI's) y beneficios para las personas con discapacidad. A nivel global del modelo DIL-D $\odot$, dicha incongruencia se refleja en la existencia de un $13.8 \%$ de empresas con niveles altos de desarrollo estratégico, pero bajo de desarrollo operativo (AB). A nivel interventivo, se nos hace del todo necesario ayudar a las organizaciones a operacionalizar la estrategia dotándola de acciones de intervención 
concreta que fomenten la implicación de todos los stakeholders. Mantener la RSC-D en un nivel estratégico sin concreción operativa se puede percibir como una acción de marketing carente de concreción y, por tanto, poco creíble.

El Modelo clasificatorio DIL-D @ contribuye al éxito de las organizaciones dotándolas de herramientas objetivas que miden el nivel de despliegue de su RSC-D, permitiéndolas crear las condiciones necesarias para que todos los individuos puedan canalizar sus habilidades, conocimientos, experiencias y motivaciones. La novedad del Modelo clasificatorio DIL-D (C) radica en que es la única herramienta desarrollada que permite medir el nivel de despliegue de la RSC-D. Además, establece las pautas que la empresa ordinaria debe seguir para la intervención en aras de promover la integración laboral, facilitando las herramientas de diagnóstico que promueven la creación de entornos integradores. 
Referencias bibliográficas

AccountAbility (20I I): AA Iooo Stakeholder Engagement Standard 201 I (en línea). <http://www.accountability.org/images/ content/3/6/362/AAioooSES\% $202010 \% 20$ PRINT.PDF $>$, acceso I7 de febrero de 2016 .

Alfaro Faus, M. y Vallés López, I. (2015): El mercado potencial de las personas con discapacidad en España. Oportunidades para la creación de valor compartido (en línea). <http:// www.convenciondiscapacidad.es/Publicaciones_ new/75_Oportunidades_Mercado.pdf $>$, acceso 9 de mayo de 2016.

Carlier, S. et al. (20I 2): "Comparing work-life balance in Spanish and Latin-American countries". European Journal of Training and Development, 36 (2): 286-307.

Carrol, A. (I979): “A three-dimensional conceptual model of corporate performance". Academy of Management Review, 4 (4): 497-505.

Comisión Europea (20II): Communication from the commission to the European Parliament, the Council, the European Economic and Social Committee and the Committee of the regions, Bruselas: Comisión Europea.

Comisión Europea (2001): Libro Verde. Fomentar un marco europeo para la responsabilidad social de las empresas, Bruselas: Comisión Europea.

Cortés Ortiz, M. (20II): La RSE en el ámbito de la discapacidad (RSE-D) (en línea). <http://www. cermi.es/es-ES/RSE-D/Lists/RSE/Attachments/4/ LA \% 20 RESPONSABILIDAD \% $20 S O C I A L$. pdf $>$, acceso 9 de mayo de 2016.

Egri, C. y Ralston, D.A. (2008): “Corporate responsibility: A review of international management research from 1998 to 2007 ". Journal of International Management, I4 (4): 319-339.

España. Ley I3/I982, de 7 de abril, de integración social de los minusválidos, Boletín Oficial del Estado, 30 de abril de I982, núm. Iо3, pp. I I IO6-I I I I 2 .
España. Real Decreto Legislativo I/20I3, de 29 de noviembre, por el que se aprueba el Texto Refundido de la Ley General de derechos de las personas con discapacidad y de su inclusión social, Boletín Oficial del Estado, 3 de diciembre de 2013, núm. 289, pp. 95635-95673.

Fligstein, N. y Feeland, R. (I995): “Theoretical and comparative perspectives on corporate organization”. Annual Review of Sociology, 2I: $2 \mathrm{I}-43$.

Foro de Expertos de Responsabilidad Social de las Empresas (2007): I, II Y III Sesión de trabajo. Definición y ámbito de la RSE, Madrid: Foro de Expertos de Responsabilidad Social de las Empresas.

Fred, D. (2003): Conceptos de Administración Estratégica, México: Pearson Education.

Fundación Bequal (20I I): Test de autoevaluación (en línea). <http://www.bequal.es/encuesta. html>, acceso I 7 de febrero de 2016.

Fundación ONCE (2009): Guía de responsabilidad social empresarial y discapacidad de la Fundación ONCE (en línea). <http://rsed. fundaciononce.es/index.html>, acceso $17 \mathrm{de}$ febrero de 2016.

Harrison, R. (1972): "Understanding your organization's character”. Harvard Business Review, 5 (3): I I9-I 28.

Hogan, G. (2003). The inclusive corporation: A disability handbook for business professionals. Ohio: University Press.

ISO (20I0): Descubriendo ISO 2600 (en línea). $<$ http://www.iso.org/iso/discovering_iso_2600oes.pdf>, acceso I7 de febrero de 2016 .

Lavrakas, P. J. (2008): Encyclopedia of survey research methods, Thousand Oaks, Ca: Sage Publications.

Lockett, A. et al. (2006): "Corporate social responsibility in management research: focus, nature, salience and sources of influence". 
Journal of Management Studies, 43 (I): II 5-I36.

Maignan, I. et al. (I999): "Corporate citizenship: Cultural antecedents and business benefits". Academy of Marketing Science Journal, 27: $455-469$.

National Disability Coordination Officer Program (s.f.): Value for Business (en línea). <http://www.ndco.stepscs.net. au/uploads/5/o/2/0/50203I7/_ndco_ valueforbusiness.pdf $>$, acceso I 7 de febrero de 2016.

Puig, M. y Martínez, A. (2008): La responsabilidad social de la Administración. Un reto para el siglo XXI (en línea). <https://wwwi.diba.cat/ uliep/pdf/fullejar/39527_fullejar.pdf>, acceso 9 de mayo de 2016 .

Quijano S. et al. (2008): “La auditoría del sistema humano (ASH) para el análisis del comportamiento humano en las organizaciones". Papeles del Psicólogo, 29: 92-106.

Quijano, S. et al. (2006): "El Modelo Global de Comportamiento Organizativo que da Soporte al ASH", en Quijano, S. (ed.): Dirección de RRHH y consultoría en las organizaciones. Barcelona: Icaria.

Romeo, M. et al. (20I6): "De la Ley de Integración Social de Minusválidos a la
Ley General de Discapacidad: Retos y oportunidades". Estudios financieros. Revista de trabajo y seguridad social: Comentarios, casos prácticos: recursos humanos, (397): 89-106.

Schein, E.H. (2010): Organizational culture and leadership (4th ed.), San Francisco: Jossey-Bass.

Servicio Estatal de Empleo (SEPE) (20I 5): Informe del Mercado de Trabajo de las Personas con Discapacidad Estatal (en línea). <https://www. sepe.es/contenidos/observatorio/mercado_ trabajo/250I-I.pdf>, acceso I7 de febrero de 2016.

Taneja, S.S. et al. (2OI I): "Researches in corporate social responsibility: A review of shifting focus, paradigms, and methodologies". Journal of Business Ethics, IоI (3): 343-364

Veríssimo, J. y Lacerda, T. (20I 5): “Does integrity matter for CSR practice in organizations? The mediating role of transformational leadership”. Business Ethics: A European Review, 24 (I): 34-5I.

Waldman, D.A. y Siegel, D. (2008): "Defining the socially responsible leader", The Leadership Quarterly, I9 (I): II7-I3I.

Werhane, P.H. et al. (1999): "Business ethics: the state of the art". International Journal of Management Reviews, I (I): I-I6. 